Information and Inference: A Journal of the IMA (2015) 4, 43-62

doi:10.1093/imaiai/iau010

Advance Access publication on 31 December 2014

\title{
Disparity and optical flow partitioning using extended Potts priors
}

\author{
XiaOHaO CAI \\ University of Cambridge, Department of Plant Sciences, Downing Street, Cambridge CB2 3EA, UK \\ JAN HENRIK Fitschen \\ University of Kaiserslautern, Department of Mathematics, Paul-Ehrlich-Str. 31, D-67663 \\ Kaiserslautern, Germany \\ Mila NiKolova \\ CMLA, ENS Cachan, CNRS, 61 Avenue du President Wilson, F-94230 Cachan, France \\ GABRIELE STEIDL ${ }^{\dagger}$ \\ University of Kaiserslautern, Department of Mathematics, Paul-Ehrlich-Str. 31, D-67663 \\ Kaiserslautern, Germany \\ ${ }^{\dagger}$ Corresponding author: Email: steidl@mathematik.uni-kl.de \\ AND \\ MARTin STORATH \\ Biomedical Imaging Group EPFL, CH-1015 Lausanne VD, Switzerland
}

[Received on 30 April 2014; revised on 15 September 2014; accepted on 19 October 2014]

\begin{abstract}
This paper addresses the problems of disparity and optical flow partitioning based on the brightness invariance assumption. We investigate new variational approaches to these problems with Potts priors and possibly box constraints. For the optical flow partitioning, our model includes vector-valued data and an adapted Potts regularizer. Using the notion of asymptotically level stable (als) functions, we prove the existence of global minimizers of our functionals. We propose a modified alternating direction method of multipliers. This iterative algorithm requires the computation of global minimizers of classical univariate Potts problems which can be done efficiently by dynamic programming. We prove that the algorithm converges both for the constrained and unconstrained problems. Numerical examples demonstrate the very good performance of our partitioning method.
\end{abstract}

Keywords: potts priors; optical flow partitioning; disparity partitioning; $\ell_{0}$ minimization; jump sparsity; ADMM-like algorithm.

\section{Introduction}

An important task in computer vision is the reconstruction of three-dimensional (3D) scenes from stereo images. Taking a photo, 3D objects are projected onto a 2D image and the depth information gets lost. If a stereo camera is used, two images are obtained. Owing to the different perspectives, there is a displacement between corresponding points in the images, which depends on the distance of the points from the camera. This displacement is called disparity and turns out to be inversely proportional to the distances of the objects, see Fig. 1 for an illustration. Therefore, disparity estimation has constituted an active research area in recent years. 

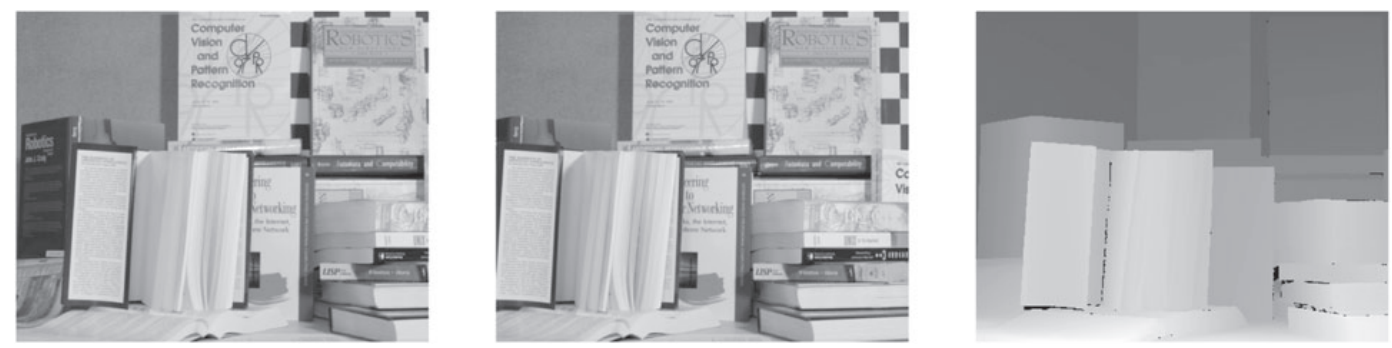

FIG. 1. Left and middle: Two images taken by a stereo camera. The shift between the images is clearly visible. Right: True disparity encoded by different gray values which shows the depth of the different objects in the scene (source: http://vision.middlebury.edu/stereo/. Last accessed December 12, 2014).

Global combinatorial optimization methods such as graph-cuts [11,39] which rely on a discrete label space of the disparity map and belief propagation $[\mathbf{3 8 , 6 8}]$ were developed as well as variational approaches $[\mathbf{1 6}, \mathbf{2 2}, \mathbf{2 6}, \mathbf{3 4}, \mathbf{4 2}, \mathbf{4 3}, \mathbf{6 4}, \mathbf{6 7}]$. In particular, in [34] the global energy function was also made convex by quantizing the disparity map and converting it into a set of binary fields. Illumination variations were additionally taken into account, e.g. in $[\mathbf{1 6}, \mathbf{1 9}]$. A stereo matching algorithm based on the curvelet decomposition was developed in [45]. With the aim of reducing the computational redundancy, a histogram-based disparity estimation method was proposed in [44]. Further, methods based on nonparametric local transforms followed by normalized cross correlation (NCC) [60] and rank-transforms [72] have been used. In this paper, we are interested in the direct disparity partitioning without a preliminary separate estimation of the disparity. Moreover, we want to avoid an initial quantization of the disparity map as necessary in graph-cut methods or in [34]. We focus on a variational approach with a linearized brightness invariance assumption to constitute the data fidelity term. The Potts prior described below will serve as a regularizing term which forces the minimizer of our functional to show a good partitioning. The partitions of a disparity map correspond typically to contiguous objects in an observed scene. The Potts model has shown to be a suitable partitioning model for disparity maps, where popular segmentation algorithms are based on graph cuts [11] and semi-global matching [36] so far.

Optical flow estimation is closely related to disparity estimation, where the horizontal displacement direction has to be completed by the vertical one. In other words, we are searching for vector fields now and have to deal with vector-valued data. Variational approaches to optical flow estimation were pioneered by Horn and Schunck [37] followed by a vast number of refinements and extensions, including sophisticated data fidelity terms going beyond the brightness $[\mathbf{8 , 1 3}, \mathbf{3 3}]$ and non-smooth regularizers, e.g. TV-like ones [2,35] including also higher-order derivatives [69-71] and non-local regularizers [66], to mention only a few of them. In general, multi-scale approaches have to be taken into account to correctly determine larger and smaller flow vectors $[\mathbf{1 , 1 2 , 2 5}$. A good overview is given in $[\mathbf{8}]$. Recent comprehensive empirical evaluations $[\mathbf{6 , 3 1}]$ show that variational algorithms yield a very good performance.

Optical flow segmentation can be done by using the estimated motion vectors in a clustering algorithm to obtain regions with similar motion vectors, see, e.g. [63]. Then regions can be grouped together for several purposes in various ways $[\mathbf{6 2 , 7 5}$. In this paper, we consider a model which partitions the optical flow without previous flow estimation using a vector-valued Potts model. A main advantage of our method is that it does not require a discrete label space and the number of labels needs not be chosen a priori. Since the data term is just based on the brightness invariance assumption, 
various flow fields (not only affine ones) can be partitioned. If the object motion is not approximately piecewise constant, the motion field may consist of several subregions which makes a subsequent merging step necessary. Concerning an object respecting flow segmentation model, we refer to [61]. Based on $[\mathbf{2 0}, \mathbf{2 1}, \mathbf{5 5}]$, the authors of [61] propose a biconvex segmentation model that segments affine motions of objects. Their model merges a weighted TV-prior, where the weights correspond to edges of one image frame, and an $\ell_{\infty}$ prior to handle the number of segments, with a data term which includes matrices for the piecewise affine motion. The proposed alternating algorithm uses an additional splitting and merging strategy in the matrix parameter minimization step. The model is not suited for non-rigid motion or for situations, where objects are merged or separated over time. For more complex methods incorporating camera motion, optical flow, depth ordering and occlusions, we also refer to [58,74].

The classical (discrete) Potts model, named after Potts [49], has the form

$$
\min _{u} \frac{1}{2}\|f-u\|_{2}^{2}+\lambda\|\nabla u\|_{0}
$$

where the discrete gradient consists of directional difference operators and $\|x\|_{0}$ denotes the number of non-zero entries of $x$. Computing a global minimizer of the multivariate Potts model appears to be NP hard $[\mathbf{1 1}, \mathbf{2 3}, \mathbf{5 9}]$. For univariate data, this problem can be solved efficiently using dynamic programming $[14,29,46,65]$. In the context of Markov random fields, the computation of the global minimizers of this kind of functionals was inaugurated by Geman and Geman [32] using simulated annealing. In [40], a deterministic continuation method to restore piecewise constant images was proposed. A stochastic continuation approach was introduced and successfully used for the reconstruction of 3D tomographic images in [50]. The method and the theory were refined in [51]. Recently, theoretical results relating the probability for global convergence and the computation speed were given in [52].

There is also a rich literature on $\ell_{0}$-regularized methods (without additional difference operator), in particular in the context of sparsity and on various (convex) relaxation methods (also for data fidelity terms with linear operators). Here, we refer to the overview in [28]. Various approximations of the $\ell_{0}$ 'norm' were used in order to guarantee that the objective function has global minimizers; see, e.g. [17], among others. Note that the local and the global minimizers of least squares regularized with the $\ell_{0}$ 'norm' were described in [47].

In this paper, we concentrate ourselves on the (non-relaxed) Potts functional. We apply the following model:

$$
\min _{u \in S} \frac{1}{2}\|f-A u\|_{2}^{2}+\lambda\|\nabla u\|_{0}
$$

where $S$ is a certain compact set, $A$ a linear operator and $\|\nabla u\|_{0}$ a 'grouped' or vector-valued prior now. We prove the existence of a global minimizer of the functional using the notion of asymptotically level stable functions [3]. For single-valued data, a completely different existence proof was given in [57]. We apply an alternating direction method of multiplier(ADMM)-like algorithm to the general Potts model (1.2). Such algorithm was proposed for the partitioning of vector-valued images for the Potts model (1.1) in [56]. It appears to be faster than current methods based on graph cuts and convex relaxations of the Potts model. In particular, the number of values of the sought-after image $u$ is not a priori restricted. Our algorithm is designed for the model (1.2) which includes non-invertible linear operators in the data fidelity term as well as constraints. In the context of wavelet frame operators (instead of gradients), another minimization method for single-valued $\ell_{0}$-regularized, constrained problems was suggested in $[41,73]$. It is based on a penalty decomposition and reduces the problem mainly to the iterative solution of $\ell_{2}-\ell_{0}$ problems via hard thresholding. Convergence to a local minimizer is shown in case of an 
invertible operator $A$. However, note that in our applications the linear operators have usually a nontrivial kernel. To the best of our knowledge, this is the first time that this kind of direct partitioning model was applied for disparity and optical flow estimation.

The remaining part of the paper is organized as follows: Our disparity and optical flow partitioning models are presented in Section 2. Section 3 provides the proof that the (vector-valued) general Potts model has a global minimizer. Then, in Section 4 an ADMM-like algorithm is suggested together with the convergence proofs for the constrained and unconstrained models. Numerical experiments are shown in Section 5. Finally, Section 6 gives points for future work.

\section{Disparity and optical flow partitioning models}

In this paper, we deal with gray-value images $f: \mathcal{G} \rightarrow \mathbb{R}$ defined on the grid $\mathcal{G}:=\{1, \ldots, M\} \times$ $\{1, \ldots, N\}$ and vector fields $u=\left(u_{1}, \ldots, u_{d}\right): \mathcal{G} \rightarrow \mathbb{R}^{d}$, where $d=1$ in the disparity partitioning problem and $d=2$ in the optical flow partitioning problem. Note that

$$
u(i, j)=\left(u_{1}(i, j), \ldots, u_{d}(i, j)\right) \in \mathbb{R}^{d}, \quad(i, j) \in \mathcal{G} .
$$

By $\nabla_{1}, \nabla_{2}$, we denote derivative operators in vertical and horizontal directions, respectively. More precisely, we will use their discrete counterparts. Among the various possible discretizations of derivative operators, we focus on forward differences

$$
\nabla_{1} u(i, j):=u(i+1, j)-u(i, j), \quad \nabla_{2} u(i, j):=u(i, j+1)-u(i, j)
$$

and assume mirror boundary conditions. Further, we will need the 'grouped' $\ell_{0}$ 'norm' for vector-valued data defined by

$$
\|u\|_{0}:=\sum_{i, j=1}^{n}\|u(i, j)\|_{0}, \quad\|u(i, j)\|_{0}:= \begin{cases}0 & \text { if } u(i, j)=0_{d} \\ 1 & \text { otherwise }\end{cases}
$$

Here, $0_{d}$ denotes the null vector in $\mathbb{R}^{d}$. If $d=1$ then $\|u\|_{0}$ is the usual $\ell_{0}$ 'componentwise norm' for vectors. For the disparity and optical flow partitioning, we will apply the $\ell_{0}$ 'norm' not directly to the vectors, but rather to $\nabla_{\nu} u_{1}$ and $\nabla_{\nu} u, v=1,2$, respectively, to penalize their spatial differences. In the disparity problem, we consider $\left\|\nabla u_{1}\right\|_{0}:=\left\|\nabla_{1} u_{1}\right\|_{0}+\left\|\nabla_{2} u_{1}\right\|_{0}$ and the optical flow problem $\|\nabla u\|_{0}:=$ $\left\|\nabla_{1} u\right\|_{0}+\left\|\nabla_{2} u\right\|_{0}$. For the later one, $\left\|\nabla_{v} u\right\|_{0}=\left\|\left(\nabla_{v} u_{1}(i, j), \nabla_{\nu} u_{2}(i, j)\right)_{(i, j)}\right\|_{0}$ uses indeed the 'grouped' version of the $\ell_{0}$ 'norm'.

REMARK 2.1 To have a convenient vector-matrix notation, we reorder images $f$ and $u_{l}, l=1, \ldots, d$ columnwise into vectors vec $f$ and vec $u_{l}$ of length $n:=N M$. We address the pixels by the index set $\mathbb{I}_{n}:=\{1, \ldots, n\}$. If the meaning is clear from the context we keep the notation $f$ instead of vec $f$. In particular, we will have $u_{l} \in \mathbb{R}^{n}$ and $u=\left(u_{1}^{\top}, \ldots, u_{d}^{\top}\right)^{\top} \in \mathbb{R}^{n d}$. After columnwise reordering the forward difference operators (with mirror boundary conditions) can be written as matrices

$$
\nabla_{1}:=I_{d} \otimes I_{M} \otimes D_{N}, \quad \nabla_{2}:=I_{d} \otimes D_{M}^{\top} \otimes I_{N},
$$


where $I_{N}$ denotes the $N \times N$ identity matrix

$$
D_{N}:=\left(\begin{array}{cccccc}
-1 & 1 & & & & \\
& -1 & 1 & & & \\
& & & \ddots & & \\
& & & & -1 & 1 \\
& & & & & 0
\end{array}\right) \in \mathbb{R}^{N, N}
$$

and $\otimes$ is the tensor (Kronecker) product of matrices.

Using the indicator function of a set $S$ defined by

$$
\iota_{S}(t)= \begin{cases}0 & \text { for } t \in S \\ \infty & \text { otherwise }\end{cases}
$$

we can address box constraints on $u$ by adding the regularizing term $\iota_{S_{\mathrm{Box}}}(u)$, where

$$
S_{\mathrm{Box}}:=\left\{u \in \mathbb{R}^{d n}: u_{\min } \leqslant u \leqslant u_{\max }\right\} .
$$

Both in the disparity and optical flow partitioning problems, we are given a sequence of images. In this paper, we focus on two images $f_{1}$ and $f_{2}$ coming from (i) the appropriate left and right images taken, e.g. by a stereo camera (disparity problem), and (ii) two image frames at different times arising, e.g. from a video (optical flow problem). Then the models rely on an invariance requirement between these images. Various invariance assumptions were considered in the literature and we refer to [8] for a comprehensive overview. Here, we focus on the brightness invariance assumption. In the disparity model, we address only horizontal displacements and consider in a continuous setting

$$
f_{1}(x, y)-f_{2}\left(x-u_{1}(x, y), y\right) \approx 0 .
$$

For the optical flow model, we assume

$$
f_{1}(x, y)-f_{2}((x, y)-u(x, y)) \approx 0, \quad u:=\left(u_{1}, u_{2}\right) .
$$

Using first-order Taylor expansions around an initial disparity $\bar{u}_{1}$, respectively, an initial optical flow estimate $\bar{u}=\left(\bar{u}_{1}, \bar{u}_{2}\right)$, gives

$$
\begin{aligned}
& \text { disp. : } f_{2}\left(x-u_{1}, y\right) \approx f_{2}\left(x-\bar{u}_{1}, y\right)-\nabla_{1} f_{2}\left(x-\bar{u}_{1}, y\right)\left(u_{1}(x, y)-\bar{u}_{1}(x, y)\right), \\
& \text { flow : } f_{2}((x, y)-u) \approx f_{2}((x, y)-\bar{u})-\left(\nabla_{1} f_{2}((x, y)-\bar{u}), \nabla_{2} f_{2}((x, y)-\bar{u})\right)(u(x, y)-\bar{u}(x, y)) .
\end{aligned}
$$

To get an initial disparity, we will use a simple block-matching approach with NCC as measure for the block similarity, following the ideas in $[\mathbf{1 6 , 6 0}]$. Then the linearized invariance requirements $(2.3)$ and (2.2) become

$$
\begin{aligned}
\text { disp. : } 0 & \approx f_{1}(x, y)-f_{2}\left(x-\bar{u}_{1}, y\right)+\nabla_{1} f_{2}\left(x-\bar{u}_{1}, y\right)\left(u_{1}(x, y)-\bar{u}_{1}(x, y)\right), \\
\text { flow }: 0 & \approx f_{1}(x, y)-f_{2}((x, y)-\bar{u})+\left(\nabla_{1} f_{2}((x, y)-\bar{u}), \nabla_{2} f_{2}((x, y)-\bar{u})\right)(u(x, y)-\bar{u}(x, y)) .
\end{aligned}
$$

Note that $f_{2}((x, y)-\bar{u})$ is only well defined in the discrete setting if $(i, j)-\bar{u}$ is in $\mathcal{G}$. Later we will see that our method to compute $\bar{u}$ really fulfills this condition, thus we can carry over the continuous 
model to the discrete setting without any modifications. Using a non-negative increasing function $\varphi$ : $\mathbb{R} \rightarrow \mathbb{R}_{\geqslant 0}$, and considering only grid points $(x, y)=(i, j) \in \mathcal{G}$ the data term for the disparity partitioning model becomes

$$
\sum_{(i, j) \in \mathcal{G}} \varphi\left(\nabla_{1} f_{2}\left(i-\bar{u}_{1}, j\right) u_{1}(i, j)-\left(\nabla_{1} f_{2}\left(i-\bar{u}_{1}, j\right) \bar{u}_{1}(i, j)+f_{2}\left(i-\bar{u}_{1}, j\right)-f_{1}(i, j)\right)\right) .
$$

In this paper, we will deal with quadratic functions $\varphi(t):=\frac{1}{2} t^{2}$. Using the notation in Remark 2.1, our partitioning models become

$$
\begin{aligned}
\text { disp. }: E_{\text {disp }}\left(u_{1}\right) & :=\frac{1}{2}\left\|A_{1} u_{1}-b_{1}\right\|_{2}^{2}+\mu \iota_{S_{\mathrm{Box}}}\left(u_{1}\right)+\lambda\left(\left\|\nabla_{1} u_{1}\right\|_{0}+\left\|\nabla_{2} u_{1}\right\|_{0}\right), \\
\text { flow }: E_{\text {flow }}(u) & :=\frac{1}{2}\|A u-b\|_{2}^{2}+\mu \iota_{S_{\mathrm{Box}}}(u)+\lambda\left(\left\|\nabla_{1} u\right\|_{0}+\left\|\nabla_{2} u\right\|_{0}\right),
\end{aligned}
$$

where $\mu \in\{0,1\}, \lambda>0,\|\cdot\|_{0}$ stands for the 'group norm' in (2.1) and

$$
\begin{aligned}
A_{1} & :=\operatorname{diag}\left(\operatorname{vec}\left(\nabla_{1} f_{2}\left(i-\bar{u}_{1}, j\right)\right)\right), \\
A & :=\left(\operatorname{diag}\left(\operatorname{vec}\left(\nabla_{1} f_{2}((i, j)-\bar{u})\right)\right), \operatorname{diag}\left(\operatorname{vec}\left(\nabla_{2} f_{2}((i, j)-\bar{u})\right)\right)\right), \\
b_{1} & :=\operatorname{vec}\left(\nabla_{1} f_{2}\left(i-\bar{u}_{1}, j\right) \bar{u}_{1}(i, j)+f_{2}\left(i-\bar{u}_{1}, j\right)-f_{1}(i, j)\right), \\
b & :=\operatorname{vec}\left(\left(\nabla_{1} f_{2}((i, j)-\bar{u}), \nabla_{2} f_{2}((i, j)-\bar{u})\right) \bar{u}(i, j)+f_{2}((i, j)-\bar{u})-f_{1}(i, j)\right) .
\end{aligned}
$$

We are looking for minimizers of these functionals.

\section{Global minimizers for Potts regularized functionals}

We want to know if the functionals in (2.4) and (2.5) have global minimizers. Both $E_{\text {disp }}$ and $E_{\text {flow }}$ are lower semi-continuous (1.s.c.) and proper functionals. When $\mu=1$, the minimization of $E_{\text {disp }}$ and $E_{\text {flow }}$ is constrained to the compact set $S_{\mathrm{Box}}$ in which case (2.4) and (2.5) have global minimizers; see, e.g. [4, Proposition 3.1.1, p. 82].

Next we focus on the case $\mu=0$. More generally, we consider for arbitrary given $A \in \mathbb{R}^{n, d n}, b \in \mathbb{R}^{n}$ and $p \geqslant 1$ functionals $E: \mathbb{R}^{d n} \rightarrow \mathbb{R}$ of the form

$$
E(u):=\frac{1}{p}\|A u-b\|_{p}^{p}+\lambda\left(\left\|\nabla_{1} u\right\|_{0}+\left\|\nabla_{2} u\right\|_{0}\right), \quad \lambda>0 .
$$

The existence of a global minimizer was proved in the case $d=1$ in [57]. Here, we give a shorter and more general proof that holds for any $d \geqslant 1$ using the notion of als functions. This wide class of functions was introduced by Auslender [3] in 2000, and since then it appeared that many problems on the existence of optimal solutions are easily solved for these functions. As usual,

$$
\operatorname{lev}(E, \lambda):=\left\{u \in \mathbb{R}^{d n}: E(u) \leqslant \lambda\right\} \quad \text { for } \lambda>\inf _{u} E(u)
$$

by $E_{\infty}$ we denote the asymptotic (or recession) function of $E$ and

$$
\operatorname{ker}\left(E_{\infty}\right):=\left\{u \in \mathbb{R}^{d n}: E_{\infty}(u)=0\right\}
$$

The following definition is taken from [4, p. 94]: an 1.s.c. and proper function $E: \mathbb{R}^{d n} \rightarrow \mathbb{R} \cup\{+\infty\}$ is said to be asymptotically level stable (als) if for each $\rho>0$, each real-valued, bounded sequence $\left\{\lambda_{k}\right\}_{k}$ 
and each sequence $\left\{u_{k}\right\} \in \mathbb{R}^{d n}$ satisfying

$$
u_{k} \in \operatorname{lev}\left(E, \lambda_{k}\right), \quad\left\|u_{k}\right\| \rightarrow+\infty, \quad \frac{u_{k}}{\left\|u_{k}\right\|} \rightarrow \tilde{u} \in \operatorname{ker}\left(E_{\infty}\right)
$$

there exists $k_{0}$ such that

$$
u_{k}-\rho \tilde{u} \in \operatorname{lev}\left(E, \lambda_{k}\right) \quad \forall k \geqslant k_{0} .
$$

If for each real-valued, bounded sequence $\left\{\lambda_{k}\right\}_{k}$ there exists no sequence $\left\{u_{k}\right\}_{k}$ satisfying (3.2), then $E$ is automatically als.

In particular, coercive functions are als. It was originally exhibited in [5] (without the notion of als functions) that any als function $E$ with $\inf E>-\infty$ has a global minimizer. The proof is also given in [4, Corollary 3.4.2]. We show that the discontinuous non-coercive objective $E$ in (3.1) is als and has thus a global minimizer.

Theorem 3.1 Let $E: \mathbb{R}^{d n} \rightarrow \mathbb{R}$ be of the form (3.1) with $p \geqslant 1$. Then the following relations hold true:

(i) $\operatorname{ker}\left(E_{\infty}\right)=\operatorname{ker}(A)$.

(ii) $E$ is als.

(iii) $E$ has a global minimizer.

Proof. (i) The asymptotic function $E_{\infty}$ of $E$ can be calculated according to [24], see also [4, Theorem 2.5.1], as

$$
E_{\infty}(u)=\liminf _{\substack{u^{\prime} \rightarrow u \\ t \rightarrow \infty}} \frac{E\left(t u^{\prime}\right)}{t} .
$$

Then

$$
\begin{aligned}
E_{\infty}(u) & =\liminf _{\substack{u^{\prime} \rightarrow u \\
t \rightarrow \infty}} \frac{(1 / p)\left\|A t u^{\prime}-b\right\|_{p}^{p}+\left\|\nabla_{1}\left(t u^{\prime}\right)\right\|_{0}+\left\|\nabla_{2}\left(t u^{\prime}\right)\right\|_{0}}{t} \\
& =\liminf _{\substack{u^{\prime} \rightarrow u \\
t \rightarrow \infty}}\left(\frac{1}{p} t^{p-1}\left\|A u^{\prime}-\frac{1}{t} b\right\|_{p}^{p}+\frac{\left\|\nabla_{1}\left(t u^{\prime}\right)\right\|_{0}+\left\|\nabla_{2}\left(t u^{\prime}\right)\right\|_{0}}{t}\right) \\
& = \begin{cases}0 & \text { if } u \in \operatorname{ker}(A), \\
+\infty & \text { if } u \notin \operatorname{ker}(A) \text { and } p>1, \\
\|A u\|_{1} & \text { if } u \notin \operatorname{ker}(A) \text { and } p=1,\end{cases}
\end{aligned}
$$

and consequently $\operatorname{ker}\left(E_{\infty}\right)=\operatorname{ker}(A)$.

(ii) Let $\left\{u_{k}\right\}_{k}$ satisfy (3.2) with $u_{k}\left\|u_{k}\right\|^{-1} \rightarrow \tilde{u} \in \operatorname{ker}(A)$ and let $\rho>0$ be arbitrarily fixed. Below we compare the numbers $\left\|\nabla_{\nu} u_{k}\right\|_{0}$ and $\left\|\nabla_{v}\left(u_{k}-\rho \tilde{u}\right)\right\|_{0}, v=1,2$. There are two options.

If $(i, j) \in \operatorname{supp}\left(\nabla_{1} \tilde{u}\right):=\left\{(i, j) \in \mathcal{G}: \tilde{u}(i+1, j)-\tilde{u}(i, j) \neq 0_{d}\right\}$, then

$$
\tilde{u}(i, j)-\tilde{u}(i+1, j)=\lim _{k \rightarrow \infty} \frac{u_{k}(i, j)-u_{k}(i+1, j)}{\left\|u_{k}\right\|} \neq 0_{d}
$$

and $\left\|u_{k}(i, j)-u_{k}(i+1, j)\right\|>0$ for all but finitely many $k$. Therefore, there exists $k_{1}(i, j)$ such that

$$
\left\|u_{k}(i, j)-u_{k}(i+1, j)-\rho(\tilde{u}(i, j)-\tilde{u}(i+1, j))\right\|_{0} \leqslant\left\|u_{k}(i, j)-u_{k}(i+1, j)\right\|_{0} \quad \forall k \geqslant k_{1}(i, j) .
$$


If $(i, j) \in \mathcal{G} \backslash \operatorname{supp}\left(\nabla_{1} \tilde{u}\right)$, i.e. $\tilde{u}(i, j)-\tilde{u}(i+1, j)=0_{d}$, then clearly

$$
u_{k}(i, j)-u_{k}(i+1, j)-\rho(\tilde{u}(i, j)-\tilde{u}(i+1, j))=u_{k}(i, j)-u_{k}(i+1, j) .
$$

Combining (3.3) and (3.4) shows that

$$
\left\|u_{k}(i, j)-u_{k}(i+1, j)-\rho(\tilde{u}(i, j)-\tilde{u}(i+1, j))\right\|_{0} \leqslant\left\|u_{k}(i, j)-u_{k}(i+1, j)\right\|_{0} \quad \forall k \geqslant k_{1}(i, j)
$$

and hence

$$
\left\|\nabla_{1}\left(u_{k}-\rho \tilde{u}\right)\right\|_{0} \leqslant\left\|\nabla_{1} u_{k}\right\|_{0} \quad \forall k \geqslant k_{1}:=\max \left\{k_{1}(i, j):(i, j) \in \mathcal{G}\right\} .
$$

In the same way, there is $k_{2}$ so that

$$
\left\|\nabla_{2}\left(u_{k}-\rho \tilde{u}\right)\right\|_{0} \leqslant\left\|\nabla_{2} u_{k}\right\|_{0} \quad \forall k \geqslant k_{2} .
$$

By part (i) of the proof we know that $A \tilde{u}=0_{n}$, which jointly with (3.5) and (3.6) implies for all $k \geqslant$ $k_{0}:=\max \left\{k_{1}, k_{2}\right\}$ that

$$
\begin{aligned}
E\left(u_{k}-\rho \tilde{u}\right) & =\frac{1}{p}\left\|A\left(u_{k}-\rho \tilde{u}\right)-b\right\|_{p}^{p}+\lambda\left(\left\|\nabla_{1}\left(u_{k}-\rho \tilde{u}\right)\right\|_{0}+\left\|\nabla_{2}\left(u_{k}-\rho \tilde{u}\right)\right\|_{0}\right) \\
& =\frac{1}{p}\left\|A u_{k}-b\right\|_{p}^{p}+\lambda\left(\left\|\nabla_{1}\left(u_{k}-\rho \tilde{u}\right)\right\|_{0}+\left\|\nabla_{2}\left(u_{k}-\rho \tilde{u}\right)\right\|_{0}\right) \\
& \leqslant \frac{1}{p}\left\|A u_{k}-b\right\|_{p}^{p}+\lambda\left(\left\|\nabla_{1} u_{k}\right\|_{0}+\left\|\nabla_{2}\left(u_{k}\right)\right\|_{0}\right)=E\left(u_{k}\right) .
\end{aligned}
$$

Hence, it follows by $u_{k} \in \operatorname{lev}\left(E, \lambda_{k}\right)$ that $u_{k}-\rho \tilde{u} \in \operatorname{lev}\left(E, \lambda_{k}\right)$ for any $k \geqslant k_{0}$. Consequently, $E$ is als.

Finally, (iii) follows directly from [4, Corollary 3.4.2].

\section{ADMM-like algorithm}

In this section, we follow an idea in [56] to approximate minimizers of our more general functionals $E_{\text {disp }}$ and $E_{\text {flow }}$. Basically, the problem is reduced to the iterative computation of minimizers of the univariate classical Potts problem for which there exist efficient solvers based on dynamic programming [29]. Here, we apply the method proposed in $[\mathbf{5 7}, \mathbf{6 5}]$. We consider

$$
\min _{u \in \mathbb{R}^{n d}}\left\{F(u)+\lambda\left(\left\|\nabla_{1} u\right\|_{0}+\left\|\nabla_{2} u\right\|_{0}\right)\right\} .
$$

Clearly, we have

$$
\begin{array}{ll}
\text { disp. }(d=1): & F(u):=\frac{1}{2}\left\|A_{1} u-b_{1}\right\|_{2}^{2}+\mu \iota_{S_{\mathrm{Box}}}(u), \quad u=u_{1}, \\
\text { flow }(d=2): & F(u):=\frac{1}{2}\|A u-b\|_{2}^{2}+\mu \iota_{S_{\mathrm{Box}}}(u), \quad u=\left(u_{1}^{\top}, u_{2}^{\top}\right)^{\top} .
\end{array}
$$

For $\mu=1$ we have a (box) constrained problem; for $\mu=0$ an unconstrained one. In [56], partitioning problems of vector-valued images with $F(u):=\frac{1}{2}\|u-b\|_{2}^{2}$ were considered. In our setting, a linear operator is involved in the data term which is not a diagonal operator in the optical flow problem, see (2.6), and in both cases (2.6) and (2.7) it has a non-trivial kernel. Further, we may have box constraints 
in addition. The minimization problem can be rewritten as

$$
\min _{u, v, w \in \mathbb{R}^{n d}}\left\{F(u)+\lambda\left(\left\|\nabla_{1} v\right\|_{0}+\left\|\nabla_{2} w\right\|_{0}\right) \text { subject to } v=u, w=u\right\} .
$$

To find an approximate (local) minimizer, we suggest the following algorithm that resembles the basic structure of an ADMM $[\mathbf{1 0}, \mathbf{3 0}]$, but with inner parameters $\eta^{(k)}$ which has to go to infinity.

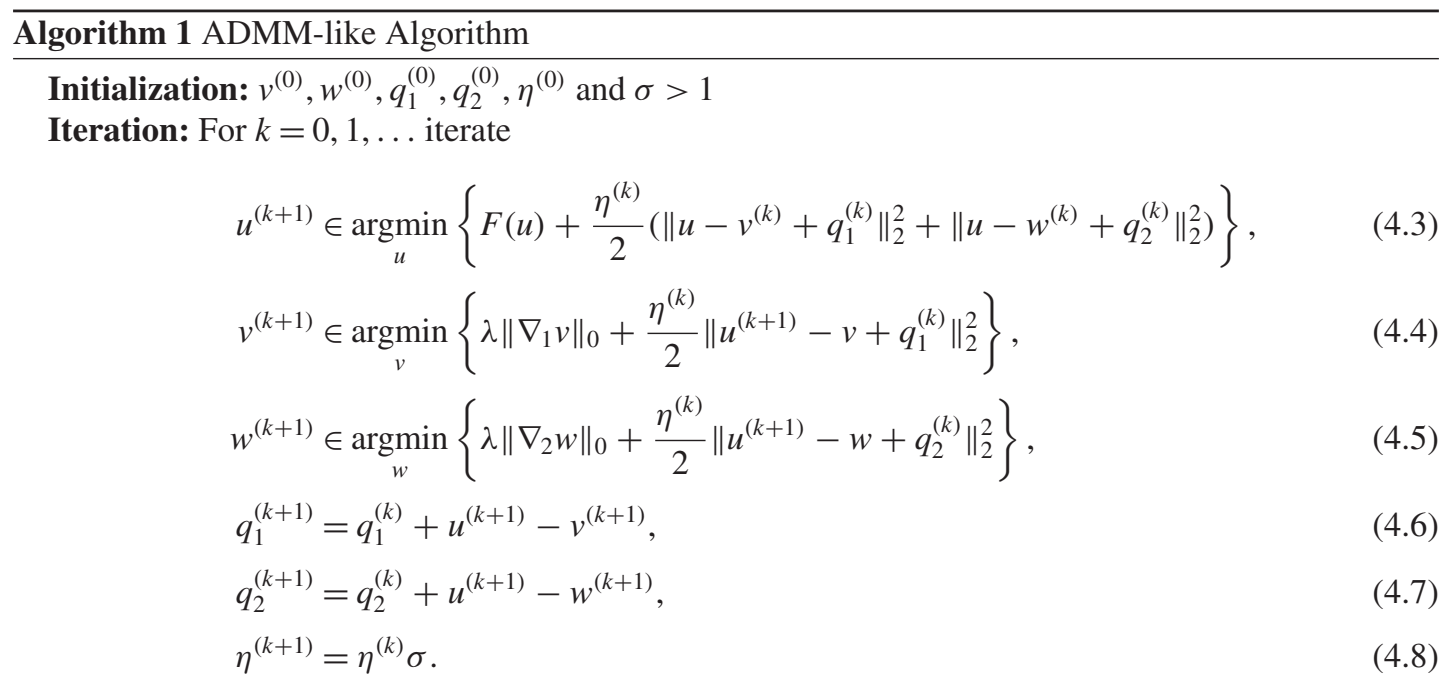

Step 1 of the algorithm in (4.3) can be computed for our optical flow term $F$ in (4.2) and $\mu=0$ by setting the gradient of the respective function to zero. Then $u^{(k+1)}$ is the solution of the linear system of equations

$$
\left(A^{\top} A+2 \eta^{(k)} I_{d n}\right) u=A^{\top} b+\eta^{(k)}\left(v^{(k)}-q_{1}^{(k)}+w^{(k)}-q_{2}^{(k)}\right) .
$$

For disparity problem (4.1), we have just to replace $A$ by $A_{1}$, which is a simple diagonal matrix and $b$ by $b_{1}$. For $\mu=1$ and the disparity problem, $u^{(k+1)}$ can be computed componentwise by straightforward computation as

$$
u^{(k+1)}=\max \left\{\min \left\{u^{(k+1 / 2)}, u_{\max }\right\}, u_{\min }\right\},
$$

where

$$
u^{(k+1 / 2)}:=\left(A_{1}^{\top} A_{1}+2 \eta^{(k)} I_{n}\right)^{-1}\left(A_{1}^{\top} b_{1}+\eta^{(k)}\left(v^{(k)}-q_{1}^{(k)}+w^{(k)}-q_{2}^{(k)}\right)\right) .
$$

For the optical flow problem and $\mu=1$, we have to minimize a box constrained quadratic problem for which there exist efficient algorithms, see, e.g. [9]. In our numerical part, the optical flow problem is handled without constraints, i.e. for $\mu=0$. In this case, only the linear system of equations (4.9) has to be solved.

The Steps 2 and 3 in (4.4) and (4.5) are univariate Potts problems which can be solved efficiently using the method proposed in $[\mathbf{5 6 , 6 5}]$. As shown in [56], the vector-valued univariate Potts problem 
can be tackled nearly in the same way as in the scalar-valued case. The arithmetic complexity is $\mathcal{O}\left(M N^{2}+N M^{2}\right)$.

Next, we prove the convergence of Algorithm 1. Owing to the NP hardness of the problem, we can in general not expect that the limit point is a (global) minimizer of the cost function. First, we deal with a general situation that involves our unconstrained problems $(\mu=0)$. We assume that any vector in the subdifferential $\partial F$ of $F$ fulfills the growth constraint

$$
u^{*} \in \partial F(u) \Rightarrow\left\|u^{*}\right\|_{2} \leqslant C\left(\|u\|_{2}+1\right) .
$$

It can be easily checked that $F: \mathbb{R}^{d n} \rightarrow \mathbb{R}^{n}$ with $F(u):=(1 / p)\|M u-m\|_{p}^{p}, p \in[1,2]$ fulfills (4.10) for any matrix $M \in \mathbb{R}^{n, d n}$ and $m \in \mathbb{R}^{n}$. Note that the variable $C$ stands for any constant in the remainder of the paper.

TheOREM 4.1 Let $F: \mathbb{R}^{d n} \rightarrow \mathbb{R} \cup\{+\infty\}$ be a proper, closed, convex function which fulfills (4.10). Then Algorithm 1 converges in the sense that $\left(u^{(k)}, v^{(k)}, w^{(k)}\right) \rightarrow(\hat{u}, \hat{v}, \hat{w})$ as $k \rightarrow \infty$ with $\hat{u}=\hat{v}=\hat{w}$ and $\left(q_{1}^{(k)}, q_{2}^{(k)}\right) \rightarrow(0,0)$ as $k \rightarrow \infty$.

Proof. By (4.6), we have

$$
\begin{aligned}
\frac{\eta^{(k)}}{2}\left\|q_{1}^{(k+1)}\right\|_{2}^{2} & =\frac{\eta^{(k)}}{2}\left\|u^{(k+1)}-v^{(k+1)}+q_{1}^{(k)}\right\|_{2}^{2} \\
& \leqslant \lambda\left\|\nabla_{1} v^{(k+1)}\right\|_{0}+\frac{\eta^{(k)}}{2}\left\|u^{(k+1)}-v^{(k+1)}+q_{1}^{(k)}\right\|_{2}^{2}
\end{aligned}
$$

and by (4.4) further

$$
\begin{aligned}
\frac{\eta^{(k)}}{2}\left\|q_{1}^{(k+1)}\right\|_{2}^{2} & \leqslant \lambda\left\|\nabla_{1}\left(u^{(k+1)}+q_{1}^{(k)}\right)\right\|_{0}+\frac{\eta^{(k)}}{2}\left\|u^{(k+1)}-\left(u^{(k+1)}+q_{1}^{(k)}\right)+q_{1}^{(k)}\right\|_{2}^{2} \\
& \leqslant \lambda\left\|\nabla_{1}\left(u^{(k+1)}+q_{1}^{(k)}\right)\right\|_{0} \\
& \leqslant \lambda n .
\end{aligned}
$$

By (4.7) and (4.5), we conclude similarly

$$
\frac{\eta^{(k)}}{2}\left\|q_{2}^{(k+1)}\right\|_{2}^{2} \leqslant \lambda n
$$

Hence, it follows

$$
\left\|q_{1}^{(k+1)}\right\|_{2}^{2} \leqslant \frac{2 \lambda n}{\eta^{(k)}} \quad \text { and } \quad\left\|q_{2}^{(k+1)}\right\|_{2}^{2} \leqslant \frac{2 \lambda n}{\eta^{(k)}}
$$

which implies $q_{1}^{(k+1)} \rightarrow 0$ and $q_{2}^{(k+1)} \rightarrow 0$ as $k \rightarrow \infty$. Further, we obtain by $u^{(k)}-v^{(k)}=q_{1}^{(k)}-q_{1}^{(k-1)}$ that

$$
\left\|v^{(k)}-u^{(k)}\right\|_{2} \leqslant\left\|q_{1}^{(k)}\right\|_{2}+\left\|q_{1}^{(k-1)}\right\|_{2} \leqslant \sqrt{\frac{2 \lambda n}{\eta^{(k-1)}}}+\sqrt{\frac{2 \lambda n}{\eta^{(k-2)}}} \leqslant 2 \sqrt{\frac{2 \lambda n}{\eta^{(k-2)}}}
$$

and analogously

$$
\left\|w^{(k)}-u^{(k)}\right\|_{2} \leqslant 2 \sqrt{\frac{2 \lambda n}{\eta^{(k-2)}}}
$$


For $\epsilon(k):=v^{(k)}-u^{(k)}-q_{1}^{(k)}+w^{(k)}-u^{(k)}-q_{2}^{(k)}$, we get by (4.11-4.12) that

$$
\begin{aligned}
\|\epsilon(k)\|_{2} & \leqslant\left\|q_{1}^{(k)}\right\|_{2}+\left\|q_{2}^{(k)}\right\|_{2}+\left\|v^{(k)}-u^{(k)}\right\|_{2}+\left\|w^{(k)}-u^{(k)}\right\|_{2} \\
& \leqslant \sqrt{\frac{2 \lambda n}{\eta^{(k-1)}}}+\sqrt{\frac{2 \lambda n}{\eta^{(k-1)}}}+2 \sqrt{\frac{2 \lambda n}{\eta^{(k-2)}}}+2 \sqrt{\frac{2 \lambda n}{\eta^{(k-2)}}} \leqslant 6 \sqrt{\frac{2 \lambda n}{\eta^{(k-2)}}},
\end{aligned}
$$

i.e. $\|\epsilon(k)\|_{2}$ decreases exponentially. By Fermat's theorem, the proximum $u^{(k+1)}$ in (4.3) has to fulfill

$$
0 \in \partial F\left(u^{(k+1)}\right)+\eta^{(k)}\left(u^{(k+1)}-v^{(k)}+q_{1}^{(k)}+u^{(k+1)}-w^{(k)}+q_{2}^{(k)}\right)
$$

so that there exists $p^{(k+1)} \in \partial F\left(u^{(k+1)}\right)$, satisfying

$$
\begin{aligned}
0 & =p^{(k+1)}+\eta^{(k)}\left(u^{(k+1)}-v^{(k)}+q_{1}^{(k)}+u^{(k+1)}-w^{(k)}+q_{2}^{(k)}\right) \\
& =p^{(k+1)}+\eta^{(k)}\left(u^{(k)}-v^{(k)}+q_{1}^{(k)}+u^{(k)}-w^{(k)}+q_{2}^{(k)}\right)+2 \eta^{(k)}\left(u^{(k+1)}-u^{(k)}\right) \\
& =p^{(k+1)}-\eta^{(k)} \epsilon(k)+2 \eta^{(k)}\left(u^{(k+1)}-u^{(k)}\right) .
\end{aligned}
$$

Rearranging terms, taking the norm and applying the triangle inequality leads to

$$
\left\|u^{(k+1)}-u^{(k)}\right\|_{2} \leqslant \frac{\left\|p^{(k+1)}\right\|_{2}}{2 \eta^{(k)}}+\frac{1}{2}\|\epsilon(k)\|_{2} .
$$

Since $\|x-y\| \geqslant\|x\|-\|y\|$ and by assumption (4.10), it follows

$$
\begin{aligned}
\left\|u^{(k+1)}\right\|_{2} & \leqslant \frac{\left\|p^{(k+1)}\right\|_{2}}{2 \eta^{(k)}}+\frac{1}{2}\|\epsilon(k)\|_{2}+\left\|u^{(k)}\right\|_{2} \\
& \leqslant \frac{C\left\|u^{(k+1)}\right\|_{2}}{2 \eta^{(k)}}+\frac{C}{2 \eta^{(k)}}+\frac{1}{2}\|\epsilon(k)\|_{2}+\left\|u^{(k)}\right\|_{2} .
\end{aligned}
$$

Since $C / 2 \eta^{(k)} \rightarrow 0$ as $k \rightarrow \infty$, there exists a $K$ such that $1<1 /\left(1-C / 2 \eta^{(k)}\right) \leqslant \tau:=\sqrt{\sigma}$ for all $k>K$. Now (4.15) implies

$$
\left\|u^{(k+1)}\right\|_{2}\left(1-\frac{C}{2 \eta^{(k)}}\right) \leqslant \frac{C}{2 \eta^{(k)}}+\frac{1}{2}\|\epsilon(k)\|_{2}+\left\|u^{(k)}\right\|_{2}
$$


which gives for $k>K$ the estimates

$$
\begin{aligned}
\left\|u^{(k+1)}\right\|_{2} & \leqslant \tau \frac{C}{2 \eta^{(k)}}+\tau \frac{1}{2}\|\epsilon(k)\|_{2}+\tau\left\|u^{(k)}\right\|_{2} \\
& \leqslant \tau \frac{C}{2 \eta^{(k)}}+\tau \frac{1}{2}\|\epsilon(k)\|_{2}+\tau^{2} \frac{C}{2 \eta^{(k-1)}}+\tau^{2} \frac{1}{2}\|\epsilon(k-1)\|_{2}+\tau^{2}\left\|u^{(k-1)}\right\|_{2} \\
& \leqslant \tau^{k+1-K}\left\|u^{(K)}\right\|_{2}+\sum_{j=1}^{k+1-K} \frac{C \tau^{j}}{2 \eta^{(k+1-j)}}+\sum_{j=1}^{k+1-K} \frac{\tau^{j}}{2}\|\epsilon(k+1-j)\|_{2} \\
& \leqslant \tau^{k+1}\left(\left\|u^{(K)}\right\|_{2}+\sum_{j=1}^{k+1-K} \frac{C}{2 \eta^{(k+1-j)}}+\sum_{j=1}^{k+1-K} \frac{1}{2}\|\epsilon(k+1-j)\|_{2}\right)
\end{aligned}
$$

and by the exponential decay of $\|\epsilon(k)\|_{2}$ with $\eta^{(k)}$ further

$$
\left\|u^{(k+1)}\right\|_{2} \leqslant C \tau^{k+1} .
$$

Using this relation together with (4.10) and (4.8) in (4.14), we conclude

$$
\begin{aligned}
\left\|u^{(k+1)}-u^{(k)}\right\|_{2} & \leqslant \frac{\left\|p^{(k+1)}\right\|_{2}}{2 \eta^{(k)}}+\frac{1}{2}\|\epsilon(k)\|_{2} \\
& \leqslant \frac{C\left\|u^{(k+1)}\right\|_{2}}{2 \eta^{(k)}}+\frac{C}{2 \eta^{(k)}}+\frac{1}{2}\|\epsilon(k)\|_{2} \\
& \leqslant \frac{C^{2} \tau^{k+1}}{2 \eta^{(k)}}+\frac{C}{2 \eta^{(k)}}+\frac{1}{2}\|\epsilon(k)\|_{2} \\
& \leqslant \frac{C^{2}}{2 \eta^{(0)} \sigma^{\frac{k-1}{2}}}+\frac{C}{2 \eta^{(k)}}+3 \sqrt{\frac{2 \lambda n}{\eta^{(k-2)}}} .
\end{aligned}
$$

Thus, $\left\|u^{(k+1)}-u^{(k)}\right\|_{2}$ decreases exponentially. Therefore, it is a Cauchy sequence and $\left\{u^{(k)}\right\}_{k}$ converges to some $\hat{u}$ as $k \rightarrow \infty$. Since $q_{1}^{(k)} \rightarrow 0$ and $q_{2}^{(k)} \rightarrow 0$ as $k \rightarrow \infty$, we obtain by (4.6) and (4.7) that $\left\{v^{(k)}\right\}_{k}$ and $\left\{w^{(k)}\right\}_{k}$ also converge to $\hat{u}$. This finishes the proof.

The assumptions in the next theorem fit to our constrained models $(\mu=1)$, but are more general.

TheOREM 4.2 Let $F: \mathbb{R}^{d n} \rightarrow \mathbb{R} \cup\{+\infty\}$ be any function that is bounded on its domain. Further assume that (4.3) has a global minimizer. Then Algorithm 1 converges in the sense that $\left(u^{(k)}, v^{(k)}, w^{(k)}\right) \rightarrow$ $(\hat{u}, \hat{v}, \hat{w})$ as $k \rightarrow \infty$ with $\hat{u}=\hat{v}=\hat{w}$ and $\left(q_{1}^{(k)}, q_{2}^{(k)}\right) \rightarrow(0,0)$ as $k \rightarrow \infty$.

Proof. As in the proof of Theorem 4.1, we can show that (4.13) holds true for $\epsilon(k):=v^{(k)}-u^{(k)}-$ $q_{1}^{(k)}+w^{(k)}-u^{(k)}-q_{2}^{(k)}$. The quadratic term in (4.3) can be rewritten as

$$
\begin{aligned}
\left\|u-v^{(k)}+q_{1}^{(k)}\right\|_{2}^{2}+\left\|u-w^{(k)}+q_{2}^{(k)}\right\|_{2}^{2} & =2\langle u, u\rangle+2\left\langle u, q_{1}^{(k)}-v^{(k)}+q_{2}^{(k)}-w^{(k)}\right\rangle+C \\
& =2\left\|u-u^{(k)}\right\|_{2}^{2}-2\langle u, \epsilon(k)\rangle+C .
\end{aligned}
$$


Thus, the first step of Algorithm 1 is equivalent to

$$
u^{(k+1)} \in \underset{u}{\operatorname{argmin}}\left\{F(u)+\eta^{(k)}\left\|u-u^{(k)}\right\|_{2}^{2}-\eta^{(k)}\langle\epsilon(k), u\rangle\right\} .
$$

This implies

$$
F\left(u^{(k+1)}\right)+\eta^{(k)}\left\|u^{(k+1)}-u^{(k)}\right\|_{2}^{2}-\eta^{(k)}\left\langle\epsilon(k), u^{(k+1)}\right\rangle \leqslant F\left(u^{(k)}\right)-\eta^{(k)}\left\langle\epsilon(k), u^{(k)}\right\rangle
$$

and further

$$
\left\|u^{(k+1)}-u^{(k)}\right\|_{2}^{2} \leqslant \frac{F\left(u^{(k)}\right)-F\left(u^{(k+1)}\right)}{\eta^{(k)}}-\left\langle\epsilon(k), u^{(k)}-u^{(k+1)}\right\rangle .
$$

Using the boundedness of $F$ and the Cauchy-Schwarz inequality leads to

$$
\left\|u^{(k+1)}-u^{(k)}\right\|_{2}^{2} \leqslant \frac{C}{\eta^{(k)}}+\|\epsilon(k)\|_{2}\left\|u^{(k)}-u^{(k+1)}\right\|_{2} .
$$

Since $\epsilon(k) \rightarrow 0$ as $k \rightarrow \infty$, we conclude that $\left\|u^{(k)}-u^{(k+1)}\right\|_{2}$ is bounded so that

$$
\left\|u^{(k+1)}-u^{(k)}\right\|_{2}^{2} \leqslant \frac{C}{\eta^{(k)}}+C\|\epsilon(k)\|_{2} .
$$

Thus, $\left\|u^{(k)}-u^{(k+1)}\right\|_{2}$ is decreasing exponentially and $\left\{u^{(k)}\right\}_{k}$ converges to some $\hat{u}$ as $k \rightarrow \infty$.

\section{Numerical results}

In this section, we present numerical results obtained by our partitioning approaches. All examples were executed on a computer with an Intel Core i7-870 Processor (8M Cache, $2.93 \mathrm{GHz}$ ) and $8 \mathrm{~GB}$ physical memory, 64 Bit Linux. Further, we have incorporated additional diagonal directions in our implementation to get a better 'rotation invariance', see [56]. More precisely, we have used the discretization

$$
\left\|\nabla_{\text {iso }} u\right\|_{0}:=(\sqrt{2}-1)\left(\left\|\nabla_{1} u\right\|_{0}+\left\|\nabla_{2} u\right\|_{0}\right)+\frac{\sqrt{2}-1}{\sqrt{2}}\left(\left\|\nabla_{1,2} u\right\|_{0}+\left\|\nabla_{2,1} u\right\|_{0}\right),
$$

where $\nabla_{1,2} u(i, j):=u(i+1, j+1)-u(i, j)$, and $\nabla_{2,1} u(i, j):=u(i+1, j-1)-u(i, j)$. We compare our direct partitioning methods (2.4) and (2.5) via Algorithm 1 with a two-stage approach consisting of (i) disparity, respectively, optical flow estimation, and (ii) partitioning of the estimated values. More precisely, the two-stage algorithm performs as follows:

(i) In the first step, the disparity is estimated using the TV regularized model

$$
\min _{u_{1} \in S_{\mathrm{Box}}}\left\{\frac{1}{2}\left\|A_{1} u_{1}-b_{1}\right\|_{2}^{2}+\iota_{S_{\mathrm{Box}}}\left(u_{1}\right)+\alpha_{1}\left\|\left|\nabla u_{1}\right|\right\|_{1}\right\},
$$

with $A_{1}$ and $b_{1}$ defined by (2.6) and (4.6), respectively. Here, $\left|\nabla u_{1}\right|$ stands for the discrete version of $\left(\left(\left(\partial u_{1} / \partial x\right)(x, y)\right)^{2}+\left(\left(\partial u_{1} / \partial y\right)(x, y)\right)^{2}\right)^{1 / 2}$, i.e. we use the isotropic ('rotationally invariant') TV version. Such model was proposed for the disparity estimation in [16] and can be found 
with, e.g. shearlet regularized $\ell_{1}$ norm in [27]. For estimating the optical flow, we minimize

$$
\min _{u}\left\{\frac{1}{2}\|A u-b\|_{2}^{2}+\alpha_{1}\left\|\sqrt{\left|\nabla u_{1}\right|^{2}+\left|\nabla u_{2}\right|^{2}}\right\|_{1}\right\},
$$

with $A$ and $b$ defined by (2.7) and (2.8), respectively. The global minimizers of the convex functionals (5.2) and (5.3) were computed via the primal-dual hybrid gradient method proposed in $[\mathbf{1 5}, \mathbf{4 8}]$. Clearly, one could use other iterative first-order (primal-dual) methods, see, e.g. [18].

(ii) In the second step, the estimated disparity, respectively, optical flow is partitioned by the method in [56] which minimizes, e.g. for the disparity the functional

$$
\min _{u_{1}}\left\{\frac{1}{2}\left\|u_{1}-u_{1, \text { est }}\right\|_{2}^{2}+\alpha_{2}\left\|\nabla_{\text {iso }} u_{1}\right\|_{0}\right\},
$$

where $u_{1, \text { est }}$ is the disparity estimated in the first step. For the approximation of a minimizer, we use the software package Pottslab http://pottslab.de. Last accessed December 12, 2014 with default parameters.

Next we comment on the direct partitioning implementation. Our partitioning models (2.4) and (2.5) are based on the knowledge of initial values $\bar{u}_{1}$ and $\bar{u}$ for the disparity, respectively, the optical flow. Here, we use a simple block-matching-based algorithm, see [16]. This method consists basically of a search within a given range. For each pixel in the first image, we compare its surrounding block with surrounding blocks of pixels in the search range of the second image. The chosen block size is $7 \times 7$. As a similarity measure, we use the NCC [60]. Finally, we apply a median filter to the initial guess to reduce the influence of outliers. Since $\left(i-\bar{u}_{1}, j\right)$, respectively $(i, j)-\bar{u}(i, j)$ are the grid coordinates of the pixel in the second image corresponding to pixel $(i, j)$ in the first image, we see that $f_{2}\left(i-\bar{u}_{1}, j\right)$, respectively, $f_{2}((i, j)-\bar{u})$ are really well-defined grid functions. The algorithm is initialized with $v^{(0)}=w^{(0)}=\bar{u}_{1}$ for the disparity partitioning and $v^{(0)}=w^{(0)}=\bar{u}$ for the flow partitioning; further $q_{i}^{(0)}, i=1,2$ are zero matrices and $\eta^{(0)}=0.01$.

REMARK 5.1 The parameter $\sigma$ should be chosen near to 1 . In all our examples, $\sigma=1.05$ appears to be a good choice. Our examples show the results after 100 iterations, where no differences to subsequently iterated images appear. If $\sigma$ approaches 1 , the convergence becomes very slow. No convergence was observed for $\sigma=1$, i.e. for constant $\eta$. If $\sigma$ becomes larger, the algorithm converges faster, but the result is very close to the initial values. A good compromise between convergence speed and a reasonable result is given by the chosen parameter.

We start with the disparity partitioning results. Figures 2 and 3 show the results for the images 'Venus' and 'Dolls'. Our direct partitioning algorithm can qualitatively compete with the two-stage algorithm. In particular, Fig. 3 provides a nice partitioning of the scene into image planes that contain objects of different distances from the camera.

Next, we present our results for the optical flow partitioning. The flow vectors are color coded with color $\simeq$ direction, brightness $\simeq$ magnitude. Figures 4 and 5 confirm that our direct method can compete with the more involved two-stage approach. Figure 6 shows the difference between the anisotropic discretization with horizontal and vertical directions, and the discretization in (5.1) which also includes the diagonal directions. We observe that the more isotropic discretization produces smoother boundaries. 

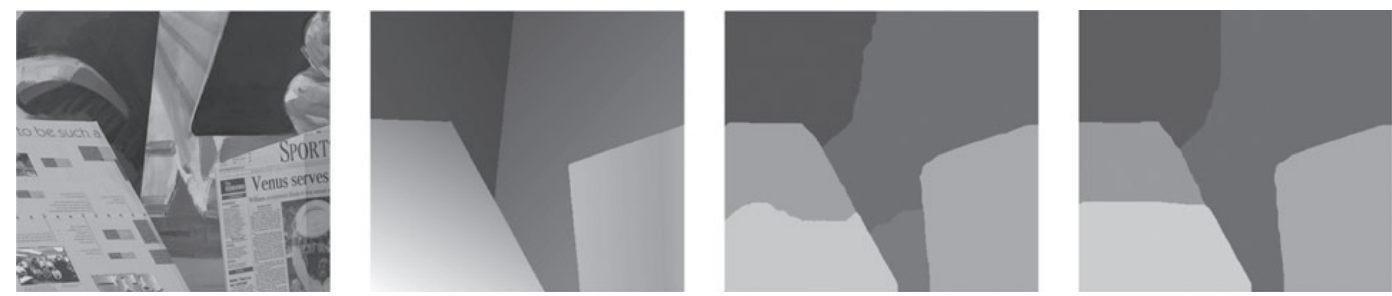

FIG. 2. Results for the images 'Venus'. Left to right: original left image, ground truth, partitioned disparity using the two-stage algorithm $\left(\alpha_{1}=0.005, \alpha_{2}=300\right)$, partitioned disparity using the direct algorithm $(\lambda=2.5)$ (source: http://vision. middlebury.edu/stereo/. Last accessed December 12, 2014 [54]).
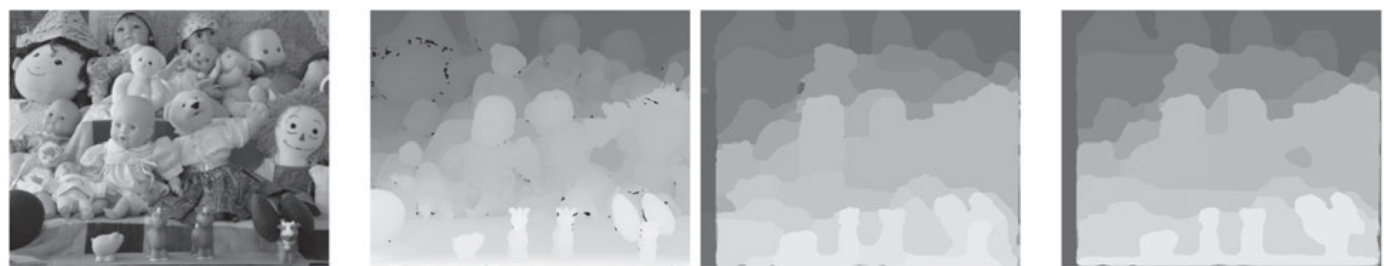

FIG. 3. Result for the images 'Dolls'. Left to right: original left image, ground truth, partitioned disparity using the two-stage algorithm $\left(\alpha_{1}=0.01, \alpha_{2}=80\right)$, partitioned disparity using the direct algorithm $(\lambda=0.8)$ (source: http://vision. middlebury.edu/stereo/. Last accessed December 12, $2014[\mathbf{5 3}])$.
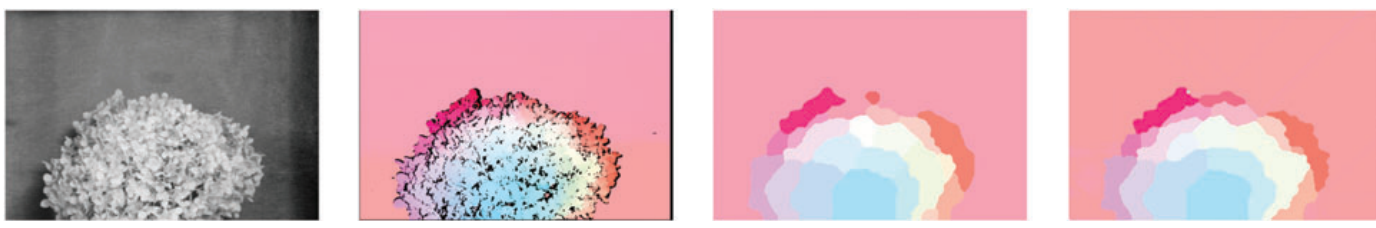

FIG. 4. Result for the images 'Hydrangea'. Left to right: first test image, ground truth, partitioned optical flow by the twostage algorithm $\left(\alpha_{1}=0.01, \alpha_{2}=35\right)$, partitioned optical flow by the direct algorithm $(\lambda=0.3)$ (source: http://vision.middlebury. edu/flow/. Last accessed December 12, 2014 [6]).
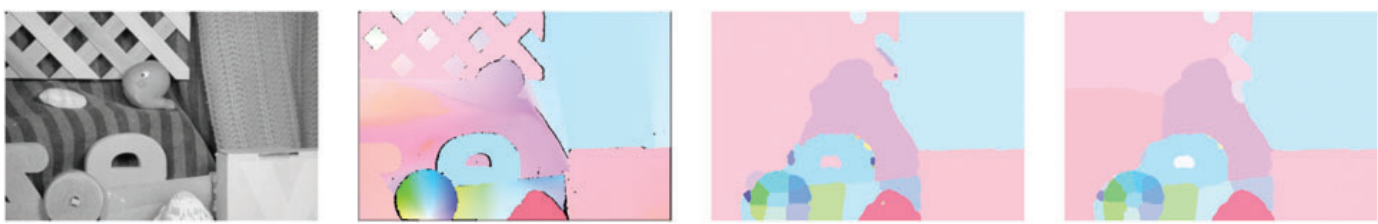

FIG. 5. Result for the images 'RubberWhale'. Left to right: first test image, ground truth, partitioned optical flow by the two-stage algorithm $\left(\alpha_{1}=0.005, \alpha_{2}=7\right)$, partitioned optical flow by the direct algorithm $(\lambda=0.12)$ (source: http://vision. middlebury.edu/flow/. Last accessed December 12, 2014 [6]).

Figure 7 shows the applicability of the proposed method for traffic surveillance. For both images, the algorithm was able to partition the main moving objects correctly. In the stroller image of Fig. 7 (right), we can see a typical effect of optical flow estimation. Owing to a reflexion of the stroller on the car at the bottom of the image, there is another partition for the motion of this reflexion. 

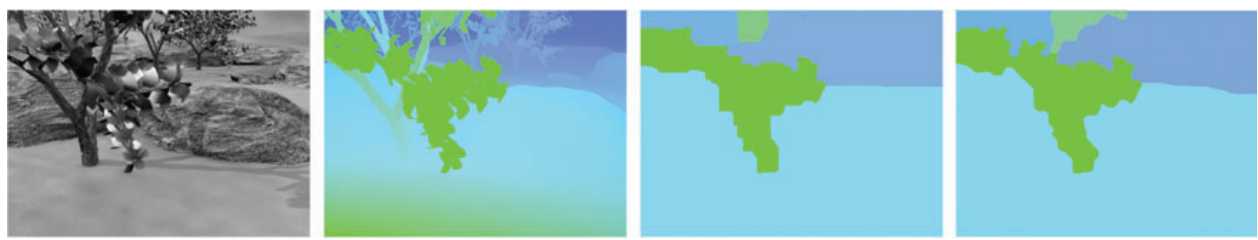

FIG. 6. Result for the images 'Grove2'. Left to right: first test image, ground truth, partitioned flow with non-isotropic and isotropic discretization $(\lambda=0.6)$ (source: http://vision.middlebury.edu/flow/. Last accessed December 12, 2014 [6]).
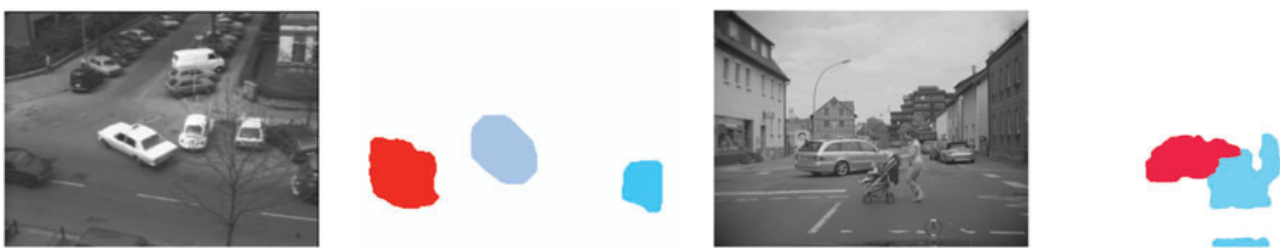

FIG. 7. Result for two traffic scenes. From left to right: image of the taxi scene (source: http://i21www.ira.uka.de/ image_sequences/. Last accessed December 12, 2014), partitioned optical flow by the direct algorithm $(\lambda=6)$ using frames 10 and 17, first image of the stroller scene (source: http://ccv.wordpress.fos.auckland.ac.nz/eisats/. Last accessed December 12, 2014 [7]), partitioned optical flow by the direct algorithm $(\lambda=2.5)$.

\section{Conclusions}

In this paper, we have proposed a new method for disparity and optical flow partitioning based on a Potts regularized variational model together with an ADMM-like algorithm. In case of the optical flow, it is adapted to vector-valued data. In this paper, we have only shown the basic approach and further refinements are planned in the future. So we intend to incorporate more sophisticated data fidelity terms. In particular, illumination changes should be handled. Towards an object-related flow, partitioning a subsequent step will be added, which takes the image frame edges and specific motions of the objects into account. This step will be adapted to the application at hand.

The crucial part for the run time of the proposed direct algorithm is the univariate Potts minimization. However, since the single problems are independent of each other, they could be solved efficiently in parallel on a GPU. Such parallel implementation is another point of future activities. Further, we want to incorporate multiple frames instead of just two in our model. From the theoretical point of view, to establish just the convergence of an algorithm to a local minimizer seems not to be enlightening, since certain constant images are contained in the set of local minimizers and we are clearly not looking for them. However, a better understanding of strict (local) minimizers and the choice of initial values for the algorithm is interesting.

\section{Acknowledgements}

Funding by the Deutsche Forschungsgemeinschaft (DFG) within the RTG GrK 1932 'Stochastic Models for Innovations in the Engineering Sciences', project area P3, is gratefully acknowledged. Some parts of the paper have been written during a visit by M. Nikolova at this Graduate School. Many thanks to M. El-Gheche (University Paris Est) for fruitful discussions on disparity estimation. 


\section{REFERENCES}

1. Anandan, P. (1989) A computational framework and an algorithm for the measurement of visual motion. Int. J. Comp. Vision, 2, 283-310.

2. Aubert, G., Deriche, R. \& Kornprobst, P. (1999) Computing optical flow via variational techniques. SiaM J. Appl. Math., 60, 156-182.

3. Auslender, A. (2000) Existence of optimal solutions and duality results under weak conditions. Math. Programm., 88, 45-59.

4. Auslender, A. \& Teboulle, M. (2003) Asymptotic Cones and Functions in Optimization and Variational Inequalities. Berlin Heidelberg: Springer.

5. Baiocchi, C., Buttazzo, G., Gastaldi, F. \& Tomarelli, F. (1988) General existence theorems for unilateral problems in continuum mechanics. Arch. Ration. Mech. Anal., 100, 149-189.

6. Baker, S., Scharstein, D., Lewis, J., Roth, S., Black, M. \& Szeliski, R. (2011) A database and evaluation methodology for optical flow. Int. J. Comp. Vision, 92, 1-31.

7. Barth, A., Siegemund, J., Meissner, A., Franke, U. \& Förstner, W. (2010) Probabilistic multi-class scene flow segmentation for traffic scenes. Pattern Recognition. Lecture Notes in Computer Science, vol. 6376. Berlin Heidelberg: Springer, pp. 503-512.

8. Becker, F., Petra, S. \& Schnörr, C. (2014) Optical flow. Handbook of Mathematical Methods in Imaging, 2nd edn. (O. Scherzer ed.). Berlin Heidelberg: Springer.

9. Bertsekas, D. P., Nedić, A. \& Ozdaglar, E. (2003) Convex Analysis and Optimization. Belmont: Athena Scientific.

10. Boyd, S., Parikh, N., Chu, E., Peleato, B. \& Eckstein, J. (2010) Distributed optimization and statistical learning via the alternating direction method of multipliers. Found. Trends Mach. Learn., 3, 1-122.

11. Bоyкov, Y., Veksler, O. \& Zabih, R. (2011) Fast approximate energy minimization via graph cuts. IEEE Trans. Pattern Anal. Mach. Intell., 23, 1222-1239.

12. Brox, T., Bruhn, A., Papenberg, N. \& Weickert, J. (2004) High accuracy optical flow estimation based on a theory for warping. Computer Vision - ECCV 2004 (T. Pajdla \& J. Matas eds). Lecture Notes in Computer Science, vol. 3024. Berlin Heidelberg: Springer, pp. 25-36.

13. Brox, T. \& Malik, J. (2011) Large displacement optical flow: descriptor matching in variational motion estimation. IEEE Trans. Pattern Anal. Mach. Intell., 33, 500-513.

14. Chambolle, A. (1995) Image segmentation by variational methods: Mumford-Shah functional and discrete approximations. SIAM J. Appl. Math., 55, 827-863.

15. Снамвolle, A. \& Роск, T. (2011) A first-order primal-dual algorithm for convex problems with applications to imaging. J. Math. Imaging Vision, 40, 120-145.

16. Chaux, C., EI-Gheche, M., Farah, J., Pesquet, J. \& Popescu, B. (2012) A parallel proximal splitting method for disparity estimation from multicomponent images under illumination variation. J. Math. Imaging Vision, 47, 1-12.

17. Chouzenoux, E., Jezierska, A., Pesquet, J.-C. \& Talbot, H. (2013) A majorize-minimize subspace approach for regularization. SIAM J. Imaging Sci., 6, 563-591.

18. Combettes, P. L. \& Pesquet, J.-C. (2010) Proximal splitting methods in signal processing. Fixed-Point Algorithms for Inverse Problems in Science and Engineering (H. H. Bauschke, R. Burachik, P. L. Combettes, V. Elser, D. R. Luke \& H. Wolkowicz eds). New York: Springer, pp. 185-212.

19. Cox, I., Roy, S. \& Hingorani., S. (1995) Dynamic histogram warping of image pairs for constant image brightness. Proceedings of International Conference on Image Process, Washington, DC, pp. 366-369.

20. Cremers, D. (2003) A variational framework for image segmentation combining motion estimation and shape regularization. IEEE Computer Society Conference on Computer Vision and Pattern Recognition, vol. 1, pp. 53-58.

21. Cremers, D. \& Soatto, S. (2005) Motion competition: a variational approach to piecewise parametric motion segmentation. Int. J. Comp. Vision, 62, 249-265. 
22. Cremers, D., Thomas, P., Kolev, K. \& Chambolle, A. (2011) Convex relaxation techniques for segmentation, stereo and multiview reconstruction. Markov Random Fields for Vision and Image Processing (A. Blake, P. Kohli \& C. Rother eds). Boston: The MIT Press.

23. Davis, G., Mallat, S. \& Avellaneda, M. (1997) Adaptive greedy approximations. Constr. Approx., 13, 57-98.

24. Dedieu, J.-P. (1977) Cônes asymptotes d'un ensemble non convexe. Application à l'optimisation. Compterendus de l'Académie des Sciences, 287, 91-103.

25. Dérian, P., HÉAs, P., Herzet, C. \& Mémin, E. (2012) Wavelet-based fluid motion estimation. Scale Space and Variational Methods in Computer Vision (A. Bruckstein, B. Haar Romeny, A. Bronstein \& M. Bronstein eds). Lecture Notes in Computer Science, vol. 6667. Berlin Heidelberg: Springer, pp. 737-748.

26. Deriche, R., Kornprobst, P. \& Aubert, G. (1996) Optical-flow estimation while preserving its discontinuities: A variational approach. Recent Developments in Computer Vision (S. Li, D. Mital, E. Teoh \& H. Wang eds). Lecture Notes in Computer Science. Berlin: Springer, pp. 69-80.

27. Fitschen, J. H. (2013) Proximal splitting methods for disparity estimation under illumination variation. Bachelor Thesis, University of Kaiserslautern.

28. Fornasier, M. \& Rauhut, H. (2014) Compressive sensing. Handbook of Mathematical Methods in Imaging, 2nd edn. (O. Scherzer ed.). New York: Springer.

29. Friedrich, F., Kempe, A., Liebscher, V. \& Winkler, G. (2008) Complexity penalized M-estimation. J. Comput. Graph. Statist., 17, pp. 201-224.

30. Gabay, D. (1983) Applications of the method of multipliers to variational inequalities. Augmented Lagrangian Methods: Applications to the Solution of Boundary Value Problems, chap. IX (M. Fortin \& R. Glowinski eds). Amsterdam: North-Holland, pp. 299-340.

31. Geiger, A., Lenz, P., Stiller, C. \& Urtasun, R. (2013) Vision meets robotics: The KiTTI dataset. Int. J. Robot. Res., 32, 1231-1237.

32. Geman, S. \& Geman, D. (1984) Stochastic relaxation, Gibbs distributions, and the bayesian restoration of images. IEEE Trans. Pattern Anal. Mach. Intell., 6, 721-741.

33. Hafner, D., Demetz, O., Weickert, J. \& Reissel, M. (2013) Is the census transform good for robust optic flow computation? Scale Space and Variational Methods in Computer Vision (A. Kuijpers, K. Bredies, T. Pock \& H. Bischof eds). Lecture Notes in Computer Science, vol. 7893. Berlin Heidelberg: Springer, pp. 210-221.

34. Hiltunen, S., Pesquet, J. C. \& Pesquet-Popescu, B. (2012) Comparison of two proximal splitting algorithms for solving multilabel disparity estimation problems. European Signal and Image Processing Conference (EUSIPCO 2012), Bucharest, Romania, pp. 1134-1138.

35. Hinterberger, W., Scherzer, O., Schnörr, C. \& Weickert, J. (2002) Analysis of optical flow models in the framework of calculus of variations. Numer. Funct. Anal. Optim., 23, 69-89.

36. Hirschmuller, H. (2008) Stereo processing by semiglobal matching and mutual information. IEEE Trans. Pattern Anal. Mach. Intell., 30, 328-341.

37. Horn, B. K. \& Schunck, B. G. (1981) Determining optical flow. Artif. Intell., 17, 185-203.

38. Klaus, A., Sormann, M. \& Karner, K. (2006) Segment-based stereo matching using belief propagation and a self-adapting dissimilarity measure. Proceedings of International Conference on Pattern Recognition, Hong Kong, vol. 3, pp. 15-18.

39. Kolmogorov, V. \& ZABIH, R. (2001) Computing visual correspondence with occlusions using graph cuts. Proceedings of the Eighth IEEE International Conference on Computer Vision, vol. 2, pp. 508-515.

40. LeClerC, Y. (1989) Constructing simple stable descriptions for image partitioning. Int. J. Comp. Vision, 3, 120-145.

41. Lu, Z. (2014) Iterative hard thresholding methods for $l_{0}$ regularized convex cone programming. Math. Programm., 147, 125-154.

42. Miled, W., Pesquet, J. \& Parent, M. (2006) Disparity map estimation using a total variation bound. The 3rd Canadian Conference on Computer and Robot Vision, Quebec, Canada, pp. 48-55.

43. Miled, W., Pesquet, J. \& Parent, M. (2009) A convex optimization approach for depth estimation under illumination variation. IEEE Trans. Image Process., 18, 813-830. 
44. Min, D., Lu, J. \& Do, M. (2011) A revisit to cost aggregation in stereo matching: How far can we reduce its computational redundancy? Proceedings of the IEEE International Conference on Computer Vision, pp. 1567-1574.

45. Mukherjee, D., WANG, G. \& Wu, Q. (2010) Stereo matching algorithm based on curvelet decomposition and modified support weights. Proceedings of the IEEE International Conference on Acoustics Speech and Signal Processing, pp. 758-761.

46. Mumford, D. \& Shah, J. (1985) Boundary detection by minimizing functionals. IEEE Conference on Computer Vision and Pattern Recognition, 17, 137-154.

47. Nikolova, M. (2013) Description of the minimizers of least squares regularized with $\ell_{0}$-norm. Uniqueness of the global minimizer. SIAM J. Imaging Sci., 6, 904-937.

48. Pock, T., Chambolle, A., Cremers, D. \& Bischof, H. (2009) A convex relaxation approach for computing minimal partitions. IEEE Conference on Computer Vision and Pattern Recognition, pp. 810-817.

49. Pоттs, R. B. (1952) Some generalized order-disorder transformations. Proc. Cambridge Philos. Soc., 48, 106109.

50. Robini, M., Lachal, A. \& Magnin, I. (2007) A stochastic continuation approach to piecewise constant reconstruction. IEEE Trans. Image Process., 16, 2576-2589.

51. Robini, M. \& Magnin, I. (2010) Optimization by stochastic continuation. SIAM J. Imaging Sci., 3, $1096-1121$.

52. Robini, M. \& Reissman, P. (2013) From simulated annealing to stochastic continuation: a new trend in combinatorial optimization. J. Global Optim., 56, 185-215.

53. Scharstein, D. \& PAL, C. (2007) Learning conditional random fields for stereo. in IEEE Computer Society Conference on Computer Vision and Pattern Recognition (CVPR 2007).

54. Scharstein, D. \& Szeliski, R. (2002) A taxonomy and evaluation of dense two-frame stereo correspondence algorithms. Int. J. Comp. Vision, 42, 7-42.

55. Schoenemann, T. \& Cremers, D. (2006) Near real-time motion segmentation using graph cuts. Pattern Recognition. Lecture Notes in Computer Science, vol. 4174. Berlin Heidelberg: Springer, pp. 455-464.

56. Storath, M. \& Weinmann, A. (2014) Fast partitioning of vector-valued images. SIAM J. Imaging Sci., 7, $1826-1852$.

57. Storath, M., Weinmann, A. \& Demaret, L. (2014) Jump-sparse and sparse recovery using Potts functionals. IEEE Trans. Signal Process., 62, 3654-3666.

58. Sun, D., Sudderth, E. B. \& Black, M. J. (2010) Layered image motion with explicit occlusions, temporal consistency, and depth ordering. Advances in Neural Information Processing Systems 23 (J. Lafferty, C. Williams, J. Shawe-Taylor, R. Zemel \& A. Culotta eds). New York: Curran Associates, Inc., pp. 22262234.

59. Tropp, J. (2006) Just relax: convex programming methods for identifying sparse signals in noise. IEEE Trans. Inform. Theory, 52, 1030-1051.

60. Tsai, D., Lin, C. \& Chen, J. (2003) The evaluation of normalized cross correlations for defect detection. Pattern Recogn. Lett., 24, 2525-2535.

61. Unger, M., Werlberger, M., Pock, T. \& Bischof, H. (2012) Joint motion estimation and segmentation of complex scenes with label costs and occlusion modeling. IEEE Conference on Computer Vision and Pattern Recognition (CVPR), 2012. IEEE, pp. 1878-1885.

62. Vazquez-Reina, A., Avidan, S., Pfister, H. \& Miller, E. (2010) Multiple hypothesis video segmentation from superpixel flows. Computer Vision - ECCV 2010 (K. Daniilidis, P. Maragos \& N. Paragios eds). Lecture Notes in Computer Science, vol. 6315. Berlin Heidelberg: Springer, pp. 268-281.

63. WAng, J. \& Adelson, E. (1994) Representing moving images with layers. IEEE Trans. Image Process., 3 , 625-638.

64. WANG, L. \& YANG, R. (2011) Global stereo matching leveraged by sparse ground control points. Proceedings of the IEEE Conference on Computer Vision and Pattern Recognition, pp. 3033-3040. 
65. Weinmann, A., Storath, M. \& Demaret, L. (2012) The L1-Potts functional for robust jump-sparse reconstruction. SIAM J. Numer. Anal., $\mathbf{5 0}$ (to appear).

66. Werlberger, M., Pock, T. \& Bischof, H. (2010) Motion estimation with non-local total variation regularization. 2010 IEEE Conference on Computer Vision and Pattern Recognition (CVPR), pp. 2464-2471.

67. Werlberger, M., Unger, M., Pock, T. \& Bischof, H. (2012) Efficient minimization of the non-local Potts model. Scale Space and Variational Methods in Computer Vision (A. Bruckstein, B. Haar Romeny, A. Bronstein \& M. Bronstein eds). Lecture Notes in Computer Science, vol. 6667. Berlin Heidelberg: Springer, pp. 314-325.

68. YAng, Q., Wang, L., YAng, R., Wang, S., LiaO, M. \& Nister, D. (2006) Realtime global stereo matching using hierarchical belief propagation. Proceedings of British Machine Vision Conference, Edinburgh, UK, pp. 989-998.

69. YuAn, J., SChNÖRr, C. \& MÉmin, E. (2007) Discrete orthogonal decomposition and variational fluid flow estimation. J. Math. Imaging Vision, 28, 67-80.

70. Yuan, J., SchnÖrR, C. \& Steide, G. (2007) Simultaneous higher order optical flow estimation and decomposition. SIAM J. Sci. Comput., 29, 2283-2304.

71. Yuan, J., SChNÖRR, C. \& STeidL, G. (2009) Convex Hodge decomposition and regularization of image flows. J. Math. Imaging Vision, 33, 169-177.

72. ZABIH, R. \& WoODFILL, J. (1994) Non-parametric local transforms for computing visual correspondence. Proceedings of European Conference on Computing and Visualization, Stockholm, Sweden, pp. 15-158.

73. Zhang, Y., Dong, B. \& Lu, Z. (2013) $l_{0}$ Minimization for wavelet frame based image restoration. Math. Comp., 82, 995-1015.

74. ZhANG, G., JiA, J., HUA, W. \& BAO, H. (2011) Robust bilayer segmentation and motion/depth estimation with a handheld camera. IEEE Trans. Pattern Anal. Mach. Intell., 33, 603-617.

75. Zitnick, C., Jojic, N. \& KAng, S. B. (2005) Consistent segmentation for optical flow estimation. Tenth IEEE International Conference on Computer Vision, vol. 2, pp. 1308-1315. 